\title{
A TOXICIDADE AGUDA DO AGROTÓXICO FIPRONIL PARA O CLADÓCERO BOSMINA FREYI DE MELO AND HEBERT, 1994
}

\author{
Lidiane Cristina da Silva ${ }^{1}$ \\ Raquel Aparecida Moreira ${ }^{2}$ \\ Odete Rocha ${ }^{3}$
}

RESUMO: O fipronil é um pesticida seletivo que atua no sistema nervoso paralisando ou matando o organismo alvo. O objetivo deste trabalho foi avaliar a toxicidade aguda do agrotóxico fipronil utilizando como organism-teste o Cladocera Bosmina freyi. Os organismos foram coletados na Represa do Broa, situada no Estado de São Paulo entre os municípios de Itirapina e Brotas. Para o teste, foram adicionadas em placas de acrílico a solução controle e as soluções-teste de fipronil nas concentrações de 0,312 mg. $\mathrm{L}^{-1}$, $0,625 \mathrm{mg} \cdot \mathrm{L}^{-1}, 1,25 \mathrm{mg} \cdot \mathrm{L}^{-1}$ e $2,5 \mathrm{mg} \cdot \mathrm{L}^{-1}$, com 4 repetições para cada uma das concentrações testadas. Foram expostos 5 indivíduos de $B$. freyi em cada cavidade. O experimento foi realizado em incubadora a $24 \pm 1{ }^{\circ} \mathrm{C}$ com fotoperíodo de 16 horas luz/8 horas escuro, por $48 \mathrm{~h}$ de exposição ao agrotóxico. A mortalidade observada foi utilizada para a determinação da $\mathrm{CL}_{50}$. Para tal cálculo, utilizou-se o programa estatístico "Trimmed Spearman-Karber Method for Estimating Median Lethal Concentrations in Toxicity Biossays". Os resultados obtidos evidenciaram que o fipronil foi moderadamente tóxico para $B$. freyi durante a exposição de $24 \mathrm{~h}$ e o valor de $\mathrm{CL}_{50}-24 \mathrm{~h}$ registrado foi de $1,5 \mathrm{mg} \mathrm{L}^{-1}$. Porém, após exposição mais prolongada, de $48 \mathrm{~h}$, o efeito da toxicidade foi maior já que mais de $50 \%$ dos indivíduos morreram na concentração mais baixa, de $0,312 \mathrm{mg} \mathrm{L}^{-1}$. Comparando-se os resultados obtidos com aqueles reportados para outros cladóceros, como as espécies do gênero Daphnia (macro-zooplâncton) conclui-se que as espécies de Bosmina (micro-zooplâncton) são mais resistentes à toxicidade deste agrotóxico do que as espécies de maior porte. Conclui-se, portanto, que a espécie Bosmina freyi pode ser utilizada como um organismo-teste

\footnotetext{
${ }^{1}$ Doutoranda em Ciências, Programa de Pós-Graduação em Ecologia e Recursos Naturais, Universidade Federal de São Carlos. lidianecris2004@yahoo.com.br.

${ }_{2}^{2}$ Mestranda em Ecologia e Recursos Naturais, Universidade Federal de São Carlos. raquel.moreira88@hotmail.com.

${ }^{3}$ Docente do Departamento de Ecologia e Biologia Evolutiva, Universidade Federal de São Carlos, UFSCar. doro@ufscar.br.
} 
para testes de toxicidade com agrotóxicos, devido à sua sensibilidade e facilidade de obtenção face à ampla ocorrência em corpos de água relativamente preservados.

Palavras-chave: Agrotóxico. Cladocera. Ecotoxicologia.

\section{INTRODUÇÃO}

Devido à crescente utilização de compostos químicos tóxicos nos mais diversos setores das atividades humanas têm ocorrido uma crescente poluição de todos os tipos de ecossistemas, mas particularmente dos sistemas aquáticos continentais. Em decorrência da conscientização da sociedade e das autoridades governamentais sobre os riscos desses compostos à saúde humana e a toda a biota aquática, inúmeros ensaios biológicos foram desenvolvidos para avaliação da toxicidade de efluentes líquidos, corpos d'água receptores e sedimentos (USEPA, 1985).

A utilização de testes de caráter ecotoxicológíco, que venham fornecer informações quanto ao efeito tóxico causado em diferentes níveis de organização dos ecossistemas por substâncias químicas nele presentes, tornam-se cada dia mais importante nas avaliações de impacto ambiental. Deste modo, a realização de testes de toxicidade tem sido incluída em programas de monitoramento, constituindo uma das análises indispensáveis no controle de fontes de poluição (CETESB, 1991).

A adoção de estudos ecotoxicológicos permite fazer predições sobre os riscos de extinção das espécies, constituindo-se uma ferramenta para a compreensão da extensão dos impactos já que os organismos vivos utilizados nos testes de toxicidade funcionam como verdadeiros "biosensores" que respondem à presença dos contaminantes. Atualmente, existem métodos padronizados de testes de toxicidade para inúmeras espécies marinhas e de água doce, como por exemplo, peixes, invertebrados e algas (CETESB, 1991).

Os testes de toxicidade sejam eles agudos ou crônicos e a determinação da faixa de sensibilidade dos organismos-teste em geral são realizados por várias razões, mas o principal objetivo é gerar dados com o intuito de prever os efeitos que substâncias químicas ou efluentes complexos podem provocar nas comunidades aquáticas naturais (Winner, 1988). 


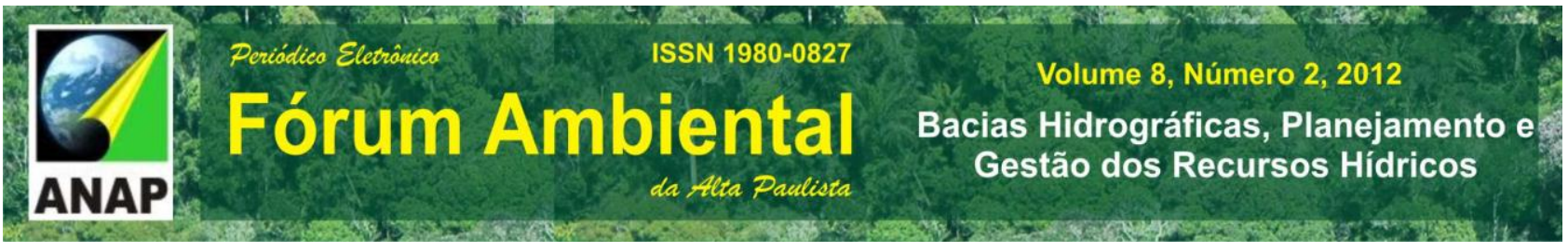

No Brasil, os organismos-teste utilizados são geralmente espécies exóticas, que, segundo Cairns (1993) são freqüentemente utilizadas para determinar a toxicidade de efluentes em ambientes aquáticos nos quais não possuem nenhuma relevância ecológica ou mesmo ocorrência. Dessa forma, são relevantes os estudos ecotoxicológicos baseados em espécies locais ou autóctones que sejam representativas de nossos ecossistemas, valendo-se da sua importância ecológica como ferramenta mais realista para definir critérios de qualidade de água e lançamentos de efluentes em corpos de água locais ou regionais.

Os cladóceros, em especial os dafinídeos, são extensivamente utilizados em testes de toxicidade aquática, pelo fato de serem organismos bem adaptados às condições de laboratório e por apresentarem uma alta sensibilidade a produtos químicos, além de exercerem um papel fundamental na comunidade aquática, a de servir como proteína animal de alto valor nutricional para peixes (Mount \& Norberg, 1984). O uso desses organismos em testes de toxicidade tem muitas vantagens, incluindo a sua facilidade de manipulação, a possibilidade de obter clones por partenogênese, seu ciclo de vida curto e a elevada sensibilidade à maioria das substâncias tóxicas (Adema 1978; Trayler e Davis 1996).

Os cladóceros mais comumente utilizados como organismos-teste em ecotoxicologia aquática são, sem dúvida as espécies Daphnia magna e Ceriodaphnia dubia, no entanto, a distribuição geográfica natural destas espécies é restrita às áreas temperadas do Hemisfério Norte (Mitchell et al. 2004).

O organismo-teste selecionado para o presente estudo, Bosmina freyi é uma espécie de Cladocera nativa facilmente encontrada em vários tipos de corpos d' água brasileiros, fato que permite sua fácil utilização em testes de toxicidade. Possui hábito principalmente litorâneo e são filtradoras de algas e protozoários que podem variar de 1 a 3 micrômetros.

$\mathrm{Na}$ seleção do agente tóxico optou-se por testar o inseticida Fipronil, considerado um pesticida seletivo que atua no sistema nervoso paralisando ou matando o organismo alvo (Rhoune-Poulenc, 1996). O Fipronil é um composto não-iônico (PIASAROLO, 2008), utilizado como produto de uso caseiro em forma de isca, para o controle de baratas, formigas, grilos e cupins (KIDD e JAMES, 1991). É utilizado também como antiparasitário 
em animais domésticos, no controle de pulgas e carrapatos. Atualmente é registrado no Brasil para o controle de cupins, besouros, lagartas e brocas nas culturas de algodão, batata, cana de açúcar, milho e soja (AGROFIT, 2007)

Desta forma, o objetivo do presente trabalho foi determinar a toxicidade deste pesticida a organismos não alvos da biota aquática e testar a sensibilidade de Bosmina freyi ao inseticida Fipronil, uma espécie abundante em corpos de água do Estado de São Paulo, ainda não testada quanto à sua adequação como organismo-teste para estudos ecotoxicológicos. Estudos desse tipo podem contribuir para a avaliação do impacto de um pesticida de uso crescente no Brasil e para a determinação da sensibilidade de uma espécie nativa, ambos constituindo subsídios para ações futuras que visem à preservação de espécies da biota aquática Neotropical.

\section{MATERIAL E MÉTODOS}

\section{Origem do material biológico}

Indivíduos da espécie Bosmina freyi De Melo and Hebert, 1994 foram coletados na Represa do Lobo, conhecida também como Represa do Broa, que está situada no Estado de São Paulo, entre os municípios de Itirapina e Brotas a $20 \mathrm{~km}$ do campus da Universidade Federal de São Carlos. Apresenta as seguintes características físicas: 7,5 $\mathrm{km}$ de comprimento máximo, 2,2 km de largura máxima e $10 \mathrm{~m}$ de profundidade máxima.

Os indivíduos foram obtidos em amostras de plâncton coletadas na referida represa por meio de arrastos verticais e horizontais combinados. A espécie foi identificada e indivíduos juvenis foram separados da amostra e submetidos ao teste. Esse estudo é um trabalho preliminar e posteriormente deverão ser realizados outros testes com a mesma espécie, porém, com clones de indivíduos adaptados às condições de cultivo. Desse modo, a faixa de sensibilidade da espécie Bosmina freyi ao fipronil e a outros compostos tóxicos de referência poderá ser determinada.

\section{Testes de toxicidade aguda}




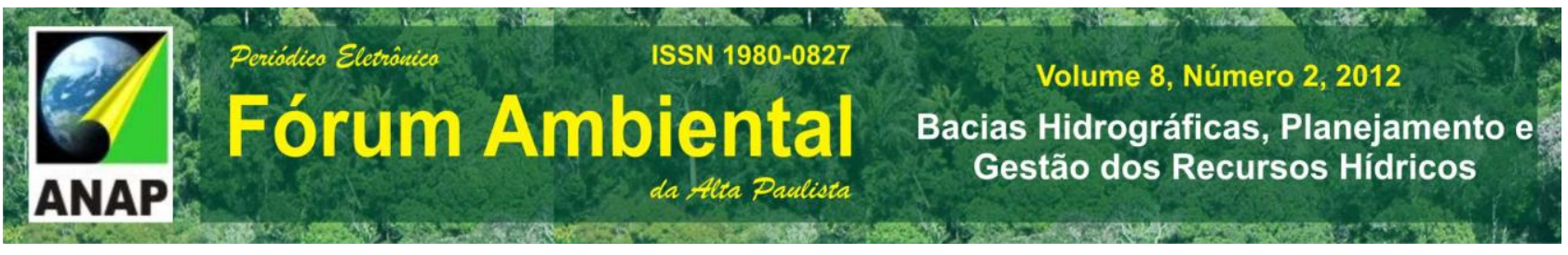

Os testes de toxicidade aguda foram feitos utilizando-se 0 produto comercial Regente 800 WG ${ }^{\circledR}(80 \%$ fipronil e $20 \%$ celulose $)$ cujo princípio ativo é o fipronil. A solução estoque foi preparada na concentração de $1 \mathrm{~g} \mathrm{~L}^{-1}$, diluído em água destilada.

Esta solução foi mantida em frasco totalmente escuro e em freezer comum $\left(-15^{\circ} \mathrm{C}\right)$ com intuito de evitar a degradação do agroquímico antes de fazer as diluições desejadas.

Em cada poço de placas de cultivo de tecido de poliestireno (Costar ${ }^{\circledR}$ ) com 12 cavidades foram adicionadas a solução controle e as soluções-teste $\left(0,312 \mathrm{mg} \cdot \mathrm{L}^{-1}, 0,625\right.$ $\mathrm{mg} \cdot \mathrm{L}^{-1}, 1,25 \mathrm{mg} \cdot \mathrm{L}^{-1}$ e $\left.2,5 \mathrm{mg} \cdot \mathrm{L}^{-1}\right)$ com 4 repetições para cada uma das concentrações. Foram utilizados cinco indivíduos da espécie Bosmina freyi em cada cavidade da placa de cultivo, distribuídos aleatoriamente em todas as cavidades. Em seguida as placas de cultivo com os organismos foram mantidas em incubadora a $24 \pm 1^{\circ} \mathrm{C}$ com fotoperíodo de 16 horas luz / 8 horas escuro com tempos de exposição ao agrotóxico de 24 e 48 horas. Ao final do teste de toxicidade aguda registrou-se o número de organismos mortos sob lupa, somando-se o número total de indivíduos mortos nas réplicas. Os resultados relativos à mortalidade observada foram utilizados para a determinação da $\mathrm{CL}_{50}$ (concentração letal para $50 \%$ dos organismos). Para tal cálculo, utilizou-se o programa estatístico "Trimmed Spearman-Karber Method for Estimating Median Lethal Concentrations in Toxicity Biossays" (Hamilton et al., 1977).

\section{RESULTADOS E DISCUSSÃO}

A tabela 1 mostra a classificação dos agrotóxicos, segundo Zucker (1985), em relação às suas classes de toxicidade. De acordo com estas o fipronil pode ser considerado moderadamente tóxico para o cladócero Bosmina freyi durante uma exposição de $24 \mathrm{~h}$, já que a $\mathrm{CL}_{50}-24 \mathrm{~h}$ registrada foi de $1,5 \mathrm{mg} \mathrm{L}^{-1}$, enquadrando-se na faixa entre 1 e $10 \mathrm{mg} \mathrm{L}^{-1}$. Porém, quando a exposição foi prolongada para o tempo de 48h, o efeito da toxicidade foi maior, indicando que o tempo de exposição do agrotóxico é um fator importante em relação aos seus efeitos. Em $48 \mathrm{~h}$ mais que $50 \%$ dos indivíduos morreram na concentração mais baixa, de $0,31 \mathrm{mg} \mathrm{L}^{-1}$. Dessa forma, não foi possível calcular a $\mathrm{CL}_{50}-48 \mathrm{~h}$. 
Nas figuras abaixo estão representados os valores da mortalidade dos indivíduos de B. freyi expostos ao Fipronil após 24h (Figura 1) e 48h (Figura 2) de exposição.

Tabela 1 - Classes de toxicidade aguda de agrotóxicos para organismos aquáticos.

\begin{tabular}{lc}
\hline Classe de toxicidade & CL50 ou CE50 (mg..' $)$ \\
\hline Extremamente tóxico & $<0,1$ \\
Altamente tóxico & $0,1 \mathrm{a} 1,0$ \\
Moderadamente tóxico & $>1,0 \mathrm{a}<10$ \\
Ligeiramente tóxico & $>10 \mathrm{a}<100$ \\
Praticamente năo-tóxico & $>100$ \\
\hline
\end{tabular}

Fonte: Zucker, 1985.

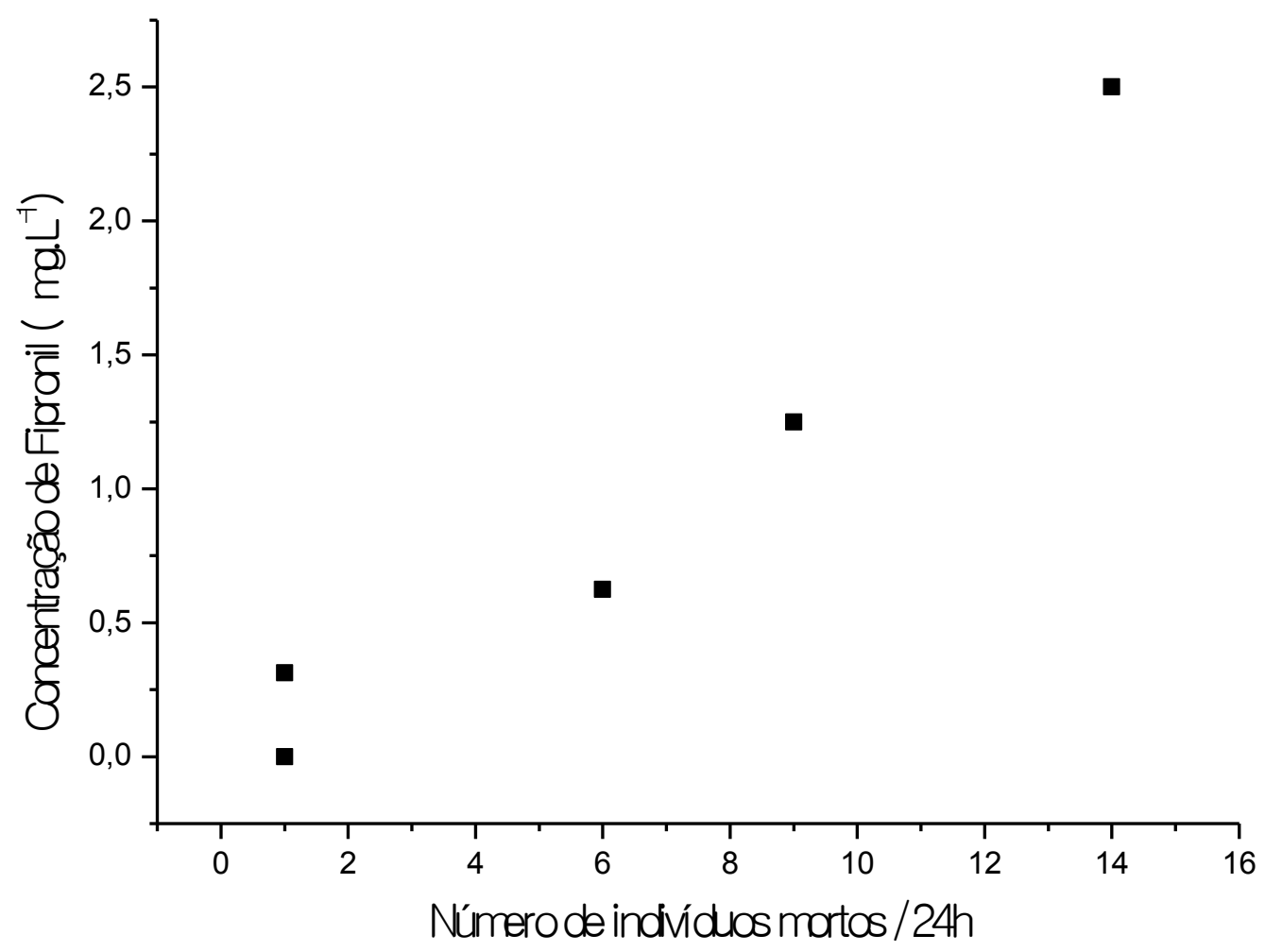

VIII Fórum Ambiental da Alta Paulista, v. 8, n.2, 2012, p. 384-394. 
Figura 1. Efeito tóxico de diferentes concentrações de Fipronil ao cladócero Bosmina freyi após $24 \mathrm{~h}$ de exposição, com intervalo de confiança de $95 \%$, teste Trimmed-SpearmannKarber (TSK).

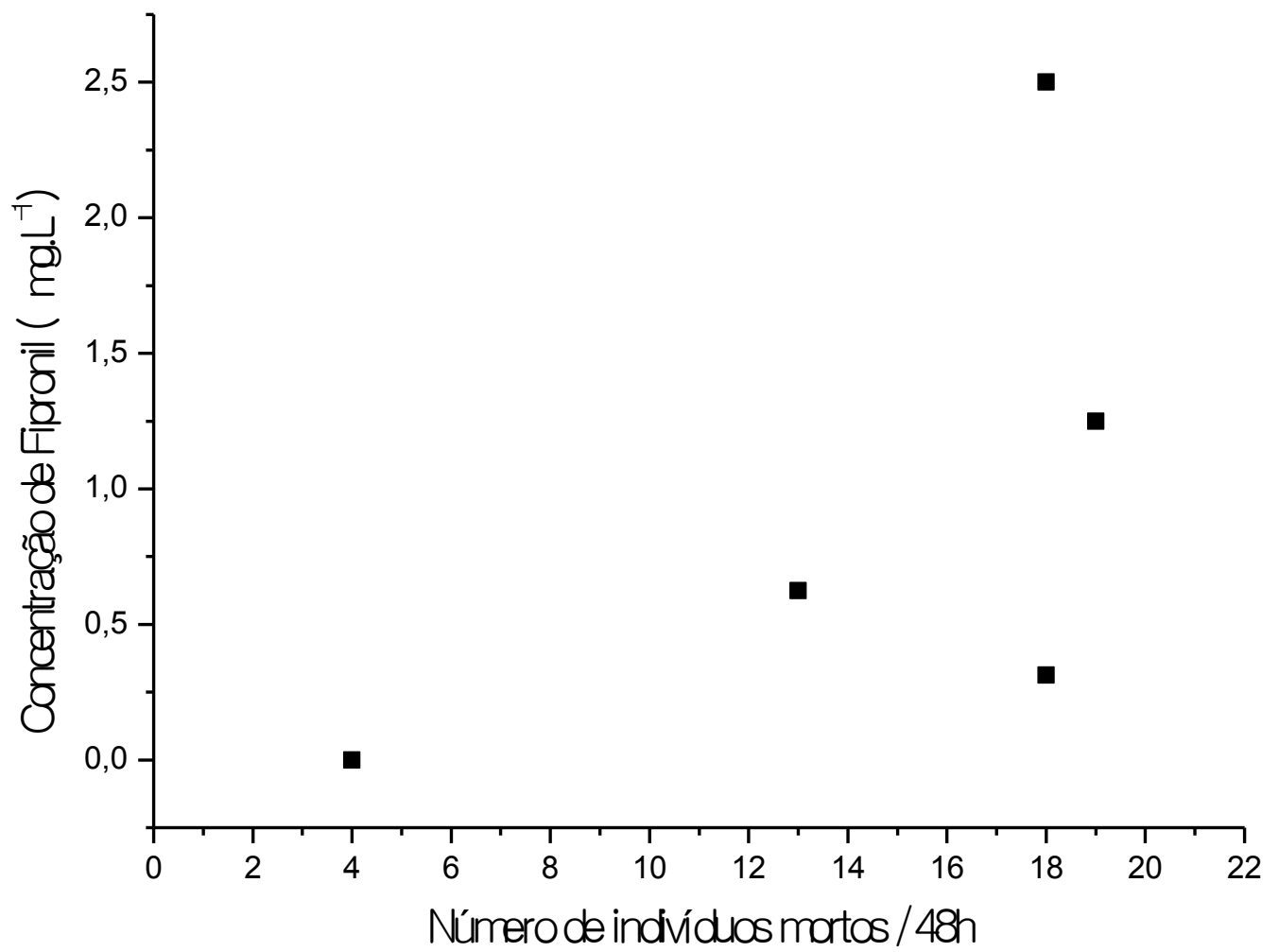

Figura 2. Efeito tóxico de diferentes concentrações de Fipronil ao cladócero Bosmina freyi após $48 \mathrm{~h}$ de exposição, intervalo de confiança de $95 \%$, teste Trimmed-SpearmannKarber (TSK).

O Fipronil é altamente tóxico para muitas espécies aquáticas e em algumas espécies é comprovada a sua bioiacumulação (Gunasekara et al, 2007). A toxicidade aguda deste pesticida foi anteriormente avaliada para alguns organismos não-alvo. Chandler et al. (2004) estimaram a $\mathrm{CL}_{50}$ - 96h de Fipronil para o copépode Amphiascus tenuiremis em $0,0068 \mathrm{mg} \cdot \mathrm{L}^{-1}$ sendo assim extremamente tóxico à essa espécie.

Scott et al. (2008) determinaram o efeito do Fipronil nos córregos usados para irrigação da cultura de arroz, por meio da avaliação da comunidade de macroinvertebrados. Verificaram que a persistência do pesticida, mesmo após seis meses 
de uma única aplicação de $0,005 \mathrm{mg} \cdot \mathrm{L}^{-1}$, diminuiu a população dos macro-invertebrados aquáticos estudados.

Entre os cladóceros, Stark et al. (2005) testaram o efeito do Fipronil para a espécie Daphnia pulex e registraram o valor de $\mathrm{CL}_{50}$ - $96 \mathrm{~h}$ de $0,016 \mathrm{mg} \cdot \mathrm{L}^{-1}$, valor este bem menor que o valor obtido para a espécie Bosmina freyi no presente estudo. Esse valor extremamente tóxico registrado pelos autores acima referidos provavelmente ocorreu devido ao maior tempo de exposição da espécie $D$. pulex ao agrotóxico (96h).

Schlenk et. al. (2001) observaram a sensibilidade do cladócero Ceriodaphnia dubia e também a elevada toxicidade do Fipronil para esta espécie, onde o valor registrado para a $\mathrm{CL}_{50}$ - 48h foi de $0,017 \mathrm{mg} \cdot \mathrm{L}^{-1}$.

O valor de $\mathrm{CL}_{50}$ registrado para a espécie Bosmina freyi em comparação com outros cladóceros sugere que as espécies pertencentes ao micro-zooplâncton possuem maior resistência quando comparadas às espécies do macro-zooplâncton. Li \& Zhou (2007) avaliaram os efeitos dos pesticidas sobre os organismos aquáticos e concluíram que sob o estresse de pesticidas, as espécies de Daphnia foram extintas, enquanto os cladóceros de pequeno porte, como Bosmina e os rotíferos tornaram-se dominantes na comunidade zooplanctônica, evidenciando assim uma maior sensibilidade pelos cladóceros de maior tamanho corporal.

Embora os resultados obtidos sejam preliminares, eles são, no entanto, promissores tendo em vista a constatação de sensibilidade da espécie Bosmina freyi ao fipronil. O fato de ser uma espécie de pequeno porte, mas relativamente resistente, levanta a hipótese da capacidade desta espécie em transformar este composto em uma forma menos tóxica, mas que em níveis sub-letais poderiam comprometer a persistência da espécie, pois organismos capazes de se desintoxicar têm que gastar energia neste processo, reduzindo provavelmente seu desempenho reprodutivo. Estes efeitos precisam ser investigados de forma mais aprofundada na busca de subsídios para a preservação da biota das águas doces brasileiras.

\section{CONCLUSÕES}


- O agrotóxico Fipronil foi tóxico ao Cladocera Bosmina freyi.

- A espécie Bosmina freyi pode ser utilizada em testes de toxicidade com agrotóxicos, podendo vir a ser um organismo-teste útil. Por ser uma espécie nativa é de grande importância sua utilização já que as espécies padronizadas atualmente são restritas a áreas temperadas.

- O tempo de exposição do agrotóxico testado é um fator importante em relação aos efeitos causados nas populações de Bosmina freyi.

- Espécies de Cladocera de pequeno porte (micro-zooplâncton) são mais resistentes à toxicidade do agrotóxico comparadas com espécies maiores (macrozooplâncton).

\section{REFERÊNCIAS}

ADEMA, D.M.M. Daphnia magna as a test animal in acute and chronic toxicity tests. Hydrobiologia. 59:125-134, 1978.

AGROFIT 2007. Ministério da Agricultura Pecuária e Abastecimento. Disponível em: <http://extranet.agricultura.gov.br/agrofi t_cons/principal_agrofi t_cons>. Acesso em: 14 fev. 2007.

CAIRNS, J. Jr. Environmental Science and Resource Management in the $21^{\text {st }}$ century: scientific perspective. Environmental Toxicology and Chemistry, v.12, p.13211329, 1993.

CETESB - COMPANHIA DE TECNOLOGIA DE SANEAMENTO AMBIENTAL. Água Teste de toxicidade aguda com Daphnia similis Claus, 1876 (Cladocera, Crustacea). São Paulo: CETESB, (Norma técnica L5 018), 1991.

CHANDLER, G. T.; CARY L. T.; VOLZ C. D.; WALSE S. S.; FERRY I. J.; KLOSTERHAUS I.S. Fipronil effects on estuarine copepod (Amphiascus tenuiremis) development, fertility, and reproduction: a rapid life-cycle assay in 96-well microplate format. Environmental Toxicology and Chemistry., v.23, n.1, p.117-124, 2004.

GUNASEKARA, A.S. et al. Fipronil behavior in environmental systems. Journal of Pesticide Science. Vol. 32, No. 3, 189-199, 2007. 
HAMILTON, M.A., RUSSO, R.C. \& THURFTON, R.B. Trimmed Spearman-Karber method for estimating median lethal concentration in toxicity bioassays.

Environmental Science \& Technology, 11 (7): 714-719, 1977.

KIDD, H. and D.R. JAMES, Eds. The Agrochemicals Handbook, Third Edition. Royal Society of Chemistry Information Services, Cambridge, UK, 1991, 3-11.

Li, S. \& Zhou, S. Side-effect of pesticides on aquatic organisms. In.: Aquatic Toxicology Research Focus. p. 79-98, 2008.

MITCHELL, S.E., HALVES, J., LAMPERT, W. Coexistence of similar genotypes of Daphnia magna in intermittent populations: response to thermal stress. Oikos 106(3):469-478, 2004.

MOUNT DI \& NORBERG, T.J. A seven-day life cycle cladoceran toxicity test. Environmental Toxicology and Chemistry, 3: 425-434, 1984.

PIASAROLO, L.; DE OLIVEIRA, R.R.L.; GUERREIRO, M.C. Influência da polaridade de pesticidas não-iônicos sobre sua sorção em um latossolo. Ciência \& Agrotecnoligia, v.32, n.6 p.1802-1809, 2008.

RHÔNE-POULENC. Fipronil Worldwide Technical Bulletin. Rhône-Poulenc Agrochimie, Lyon, France. 19 pp., 1996.

SCHLENK, D., HUGGETT, D. B., BENNETT, E., RIMOLDI, J., BEELER, A. B., BLOCK, D., HOLDER, A. W., HOVINGA, R. AND BEDIENT, P. Archives of

Environmental Contamination and Toxicology, v.41, 325-332, 2001.

SCOTT, V. M.; STEPHEN, D. P.; DENNIS, K. D. Influence of fipronil compounds and rice cultivation land-use intensity on macroinvertebrate communities in streams of southwestern Louisiana, USA. Environmental Pollution, v.152, p.497-503, 2008.

SCHLENK, D.; HUGGETT, D. B.; BENNETT, E.; RIMOLDI, J.; BEELER, A. B.; BLOCK, D.; HOLDER, A. W.; HOVINGA, R. AND BEDIENT, P. Archives of

Environmental Contamination and Toxicology, v. 41, 325-332, 2001.

STARK, D. J.; VARGAS I. R. Toxicity and hazard assessment of fipronil to Daphnia pulex. Ecotoxicology Environmental. Safety. v.62, p.11-16, 2005.

TRAYLER, K.M., DAVIS, J.A. Sensitivity of Daphnia carinata sensu lato to the insect growth regulator, pyriproxyfen. Ecotoxicology Environmental. Safety, 33(2):154-156, 1996.

USEPA - UNITED STATES ENVIRONMENTAL PROTECTION AGENCY. USEPA/600/4 85/014. Short term methods for estimating the chronic toxicity of effluents and receiving waters to freshwater organisms. Cincinnati, Ohio, 162p., 1985. 
WINNER, R.W. Evaluation on the relative sensitivities of 7.D. Daphnia magna and Ceriodaphnia dubia toxicity tests for cadmium and sodium pentachlorophenate. Environmental Toxicology and Chemistry, v.7:153-159p, 1988.

ZUCKER, E. Hazard Evaluation Division - Standard Evaluation Procedure - Acute toxicity test for freshwater fish. (USEPA Publication 540/9-85-006), 1985. 